\title{
Study of Attention-Deficit Hyperactivity Disorder (ADHD) in Children- Applying Association Rule Mining (ARM) (Survey)
}

\author{
Neema.H.N ${ }^{1}$, Dr. N.V.Balaji ${ }^{2}$ \\ Research Scholar, Department of CA, CS \& IT, Karpagam Academy of Higher Education, Coimbatore, India ${ }^{1}$ \\ Dean-Training and Placement, Dept. of CA,CS \& IT, Karpagam Academy of Higher Education, Coimbatore, India ${ }^{2}$
}

\begin{abstract}
Attention Deficit Hyperactivity Disorder (ADHD) is one of the most thoroughly researched disorders in medicine. Although many data mining techniques have been applied to diagnose the main causes of ADHD, but only few sets of clinical risk factors are considered. So the results produced by such techniques may not represent appropriate ADHD pattern and risk factors appropriately. Environmental factors also play a role in elucidating this disorder. In this study, we have designed a system that can efficiently discover the rules and the risk level of patients based on the given parameter about this disorder, comfortable with making the diagnosis and treating them with ADHD. We consider the relationship among comorbidities of ADHD based on association rule mining (ARM) among these data mining techniques with the help of Apriori Algorithm. The intention of this project is to diagnose the symptoms(patterns) that may result in ADHD in children in the earlier stages by using the Data Mining techniques .
\end{abstract}

Keywords: ADHD, Data mining, R software, Association Rule Mining, Apriori Algorithm

\section{INTRODUCTION}

Attention Deficit Hyperactivity Disorder (ADHD) is a neurobehavioral disorder found more commonly, but not exclusively, in children. Attention deficit hyperactivity disorder (ADHD) is characterized by pervasive and impairing symptoms of inattention, hyperactivity, and impulsivity according to DSM-IV. It has been associated with a broad range of negative outcomes for affected subjects and with a serious financial burden to families and society, which characterizes it as a major public health problem. The presence of ADHD in children has been known to give negative effects for many aspects of life, such as self-esteem, academic performance, social functions, and parent child relationships. By definition, the onset of hyperkinetic disorder must be present in at least two out of three environments (home, school), this behaviour must be maladaptive and pervasive. The onset of hyperkinetic disorder is before the age of 7 years, but is frequently recognised in children less than 2 years old. DSM V is proposing to keep ADHD as one of its disorders. The main proposed change is around broadening the age of onset "on and before age 12".

The causes of ADHD are not fully understood; however, several environmental and genetic risk factors have been proposed It has been suggested that dietary interventions such as $\omega-3$ FA and vitamin and mineral supplementation, restriction diets, and the avoidance of synthetic food colour additives might affect ADHD symptoms. Recently a number of studies have proposed that vitamin D might play a role in ADHD pathogenesis. A recent study proposed that vitamin $\mathrm{D}$ directly up regulates expression of tyrosine hydroxylase by binding to the nuclear vitamin receptor. It is also suggested that this vitamin is involved in the synthesis of serotonin in the brain.

\section{DATA MINING}

Data mining tools help in analytical methodology for detecting valuable information. Data Mining provides several benefits in health industry. Data mining, also known as knowledge discovery in databases, is such a research area to extract implicit, understandable, previously unknown and potentially useful information from data. Data mining helps to extract important data from a large database. It is the process of sorting through large amounts of data and picking out relevant information through the use of certain sophisticated algorithms. Data mining techniques are the result of a long process of research and product development.

A brief summarization of various data mining algorithms used for classification, clustering, and association as well as their respective advantages and drawbacks is also presented. We introduce how data mining technology that is association rule mining have been used for a multitude of purposes, including research in the healthcare field. Data 
Vol. 8, Issue 6, June 2019

mining can uncover new biomedical and healthcare knowledge for clinical and administrative decision making as well as generate scientific hypotheses from large experimental data, clinical databases, and/or biomedical literature.

A discussion of the technologies available to enable the prediction of ADHD symptoms, diagnosis and prognosis, and the discovery of hidden biomedical and healthcare patterns from related databases is offered along with a discussion of the use of data mining. Data mining is the process of finding correlations or patterns among dozens of fields in large relational databases. We have to collect data from different verticals of children such as, medical history, family history of ADHD, nature of food habit etc. All these data together forms our experimental data.

\section{R SOFTWARE}

$\mathrm{R}$ is a free software environment for statistical computing and graphics. It runs on Windows, Linux and MacOS. $\mathrm{R}$ is widely used in academia and research, as well as industrial applications. $\mathrm{R}$ is a programming language and free software environment for statistical computing and graphics that is supported by the R Foundation for Statistical Computing. The $\mathrm{R}$ language is widely used among statisticians and data miners for developing statistical software and data analysis.

\section{ASSOCIATION RULE LEARNING}

Association rule learning is a popular and well researched method for discovering interesting relations between variables in large databases in data mining. It is intended to identify strong rules discovered in databases using different measures of interestingness. Based on the concept of strong rules, Rakesh Agrawal et al. A typical and widely-used example of association rule mining is Market Basket Analysis.

\section{APRIORI ALGORITHM}

This algorithm is an algorithm is used for frequent item set mining and association rule learning. The algorithm use a level-wise search, where k-item sets (An item set which contains $\mathrm{k}$ items is known as k-item set) are used to explore $(\mathrm{k}+1)$-item sets, to mine frequent item sets from transactional database for Boolean association rules. In this algorithm, frequent subsets are extended one item at a time and this step is known as candidate generation process. Then groups of candidates are tested against the data. It identifies the frequent individual items in the database and extends them to larger and larger item sets as long as those item sets appear sufficiently often in the database.

\section{REVIEW OF LITERATURE}

A literature review reveals many results on ADHD carried out by different methods and materials of this mental disorder problem. Many people have developed various prediction models using data mining to predict ADHD.

Mr.Kati Heinonen.Ms.Katri Räikkönen and Ms Anu-Katriina Pesonen published a paper Behavioural symptoms of attention deficit/hyperactivity disorder in preterm and term children born small and appropriate for gestational age. A longitudinal study on regional birth cohort study comprising 1535 live-born infants between 03/15/1985 and 03/14/1986 admitted to the neonatal wards and 658 randomly recruited non-admitted infants, in Finland. The present study sample comprised 828 children followed up to 56 months. The association between birth status and parent-rated ADHD symptoms of the child was analysed with multiple linear and logistic regression analyses. Neither prematurity (birth $<37$ weeks of gestation) nor lower gestational age was associated with ADHD symptoms. However, small for gestational age (SGA<-2 standard deviations [SD] below the mean for weight at birth) status and lower birth weight SD score were significantly, and independently of gestational age, associated with higher ADHD symptoms. Those born SGA, relative to those born AGA, were also 3.60-times more likely to have ADHD symptoms scores above the clinical cut-off. The associations were not confounded by factors implicated as risks for pregnancy and/or ADHD.

Mr.Yueh-Ming Tai and Mr. Hung-Wen chiu published a paper about comorbidity study of ADHD : Applying assosciation rule mining to National Health Insurance of Taiwan. This paper intends to apply ARM to explore the labyrinthian network of ADHD comorbidity, and examine the practicality of ARM in comorbidity studies using clinical databases. From clinic records of Taiwan NHI,18321 youngsters aged 18 or less with diagnosis of ADHD in 2001 were recruited as case group in this study. And all their clinic diagnoses made from 2000 to 2002, as comorbidity, were categorized according to "The International Classification of Disease, $9^{\mathrm{TH}}$ Revision, Clinical Modification" diagnosis system .ARM was done with Apriori algorithm to examine the strengths of associations among those diagnoses. The support and confidence values of ARM results were examined. 
Vol. 8, Issue 6, June 2019

Mr. Leejin KIM and Mr.Sungmin Myoung published a paper Comorbidity Study of Attention-deficit Hyperactivity Disorder (ADHD) in Children: Applying Association Rule Mining (ARM) to Korean National Health Insurance Data .In this paper the highest association between ADHD and comorbid diseases was tic disorders, which is compatible with previous neurologic and epidemic studies. Our study also demonstrated the usefulness of ARM in ADHD comorbidity study in a large-scale, nationally representative dataset, namely the Korean NHID. The result of this study will be helpful to researchers evaluating the stability of their diagnosis with ADHD, and still, need some further evidence for establishing causal relationships.

Future research focuses on experiment with summarization of ARM results into a predictive model such as Bayesian network or decision tree model. The most prevalent comorbid psychiatric disorder (Table 1) of ADHD youths was mood/affective disorders group $32.34 \%$, followed by anxiety disorders group $20.98 \%$, emotional disorders with onset specific to childhood $17.76 \%$, tic disorders $16.17 \%$, conduct disorders $10.37 \%$, mild mental retardation $7.91 \%$, pervasive developmental disorders $6.37 \%$, specific developmental disorders of speech and language $5.40 \%$, mixed disorders of conduct and emotions $4.68 \%$ and specific developmental disorders of scholastic skills $3.05 \%$.

In the paper Children and ADHD: seeking control within the constraints of diagnosis ,_Brady, G.M proposed This paper focuses on a group of children who are often subject to pharmacological intervention, namely, those that are diagnosed with the condition of Attention Deficit Hyperactivity Disorder (ADHD). The contemporary and most dominant framework of understanding of behaviour associated with the condition is the Diagnostic and Statistical Manual of Mental Disorders (the most recent versions being DSM IV, 1994; DSM TR 2000, DSM V, 2013, APA). DSM V details the areas of difficulty identified as significant - in attention, impulsivity and over-activity.

Whilst a range of professionals from health, education and social care can be involved in supporting children who are so diagnosed, multiagency working and pervasive views of children's development have led to the increasing dominance of biomedical understandings across all other disciplines. The biomedical framework has become embraced by a wide range of professionals and practitioners, including psychiatrists, psychologists, teachers, (Timimi and Radcliffe, 2005), social workers, early years' workers and, increasingly, many of those who have a remit for working with children. The purpose of this paper is to contribute to knowledge about children's understandings and experiences of ADHD, drawing on a study of children in England carried out in 2000-01.

Over the years since this study was carried out clinical practice and treatment, the prevalence of cases as well as public discourses on ADHD and other neuro-psychiatric conditions may have changed, to some extent. However, it is reasonable to argue that fundamental aspects of children's understandings and experiences of living with ADHD have not changed so quickly. Increasingly, there are more children diagnosed with ADHD and prescribed medication, and yet there is still minimal attention paid to the child's perspective and the meanings which they give to living with such a diagnosis. Taken together, studies from different periods of time can contribute to our stock of knowledge about the meaning of ADHD. This can help in thinking about past, present and future generations of children and their experiences of psychiatrization.

Guilherme Polanczyk, Maurício SilvadeL ima, Bernardo Lessa Horta, Joseph Biederman,,Luis Augusto Rohde, proposed a paper The Worldwide Prevalence of ADHD: A Systematic Review and Meta regression Analysis. In this paper The worldwide prevalence estimates of attention deficit hyperactivity disorder (ADHD)/hyperkinetic disorder (HD) are highly heterogeneous. For their study. The authors searched MEDLINE and PsycINFO databases from January 1978 to December 2005 and reviewed textbooks and reference lists of the studies selected.

Authors of relevant articles from North America, South America, Europe, Africa, Asia, Oceania, and the Middle East and ADHD/HD experts were contacted. Surveys were included if they reported point prevalence of ADHD/HD for subjects 18 years of age or younger from the general population or schools according to DSM or ICD criteria. The literature search generated 9,105 records, and 303 full-text articles were reviewed. One hundred and two studies comprising 171,756 subjects from all world regions were included. Our findings suggest that geographic location plays a limited role in the reasons for the large variability of ADHD/HD prevalence estimates worldwide. Instead, this variability seems to be explained primarily by the methodological characteristics of studies.

All the above researchers have been successful in analysing the ADHD disorder data set and they developed good prediction methods. But all these tools or methods are considered only minimum parameters. Here the proposed method, which handles multidimensional parameters. It contains structured and unstructured data or multidimensional data. 
Vol. 8, Issue 6, June 2019

\section{CONCLUSION}

Over the past years, ADHD has been studied closely and much research has been carried out, particularly to elucidate the aetiology of the condition, to make a more accurate and timely diagnosis, and for effective treatments to be made available. However, much still remains to be known. This study reveals an important role of diagnosing ADHD like psychiatric diseases that is compatible with results of previous neurologic and other studies at the beginning stages. We also demonstrated the practicality of ARM in ADHD comorbidity study within an enormous database with the help of Apriori algorithm.

\section{REFERENCES}

[1]. Early Identification of Diseases Based on Responsible Attribute Using Data Mining

[2]. Mr. Sudhir M. Gorade1, Prof. Ankit Deo2, Prof. Preetesh Purohit3

[3]. ADHD: from childhood into adulthood ;Nigel Camilleri, Samer Makhoul

[4]. Children and ADHD: seeking control within the constraints of diagnosis; Brady, G.M. (2014), volume 28 (3): 218-230, DOI 10.1111/chso. 12069

[5]. Comorbidity Study of Attention-deficit Hyperactivity Disorder (ADHD) in Children: Applying Association Rule Mining (ARM) to Korean National Health Insurance Data

[6]. Leejin KIM 1 , * Sungmin MYOUNG 2

[7]. Comorbidity Study of ADHD: Applying Association Rule Mining (ARM) to National Health Insurance Data base of Taiwan; YuehMing,Hung-Wen Chiu

[8]. The Worldwide Prevalence of ADHD: A Systematic Review and Meta regression Analysis., Guilherme Polanczyk, M.D.,Maurício Silva de Lima, M.D., Ph.D. Bernardo Lessa Horta, M.D., Ph.D.,Joseph Biederman, M.D.,Luis Augusto Rohde, M.D., Ph.D

\section{BIOGRAPHIES}

Mrs. Neema.H.N, Research Scholar, Department of CA, CS \& IT, Karpagam Academy of Higher Education, Coimbatore and Assistant Professor, Department of Computer Science, Al Ameen Arts \& Science College, Ernakulam.

Dr. N.V.Balaji, Dean-Training and Placement, and Research Guide, Department of CA, CS \& IT, Karpagam Academy of Higher Education, Coimbatore. 